\title{
Integrative Systems Biology Approaches to Identify Potential Biomarkers and Pathways of Cervical Cancer
}

\author{
Arafat Rahman Oany ${ }^{1,2}$, Mamun Mia ${ }^{1,3}$, Tahmina Pervin ${ }^{4}$, Salem Ali Saleh Alyami ${ }^{5}$ \\ Mohammad Ali Moni ${ }^{* *}$
}

${ }^{1}$ Department of Biotechnology and Genetic Engineering, Faculty of Life Science, Mawlana Bhashani Science and Technology University, Tangail-1902, Bangladesh

${ }^{2}$ Aristopharma Limited, Bangladesh

${ }^{3}$ RT-PCR Laboratory, AICHI Hospital Ltd., Dhaka-1230, Bangladesh

${ }^{4}$ Biotechnology and Genetic Engineering Discipline, Life Science School, Khulna University, Khulna, Bangladesh.

${ }^{5}$ Department of Mathematics and Statistics, Faculty of Science, Imam Mohammad Ibn Saud Islamic University (IMSIU), Riyadh 13318, Saudi Arabia

${ }^{6}$ WHO Collaborating Centre on eHealth, UNSW Digital Health, School of Public Health and Community Medicine, Faculty of Medicine, UNSW Sydney, Australia.

\section{* Corresponding Author}

Mohammad Ali Moni, PhD (Cantab)

WHO Collaborating Centre on eHealth, UNSW Digital Health, School of Public Health and Community Medicine, Faculty of Medicine, UNSW Sydney, Australia

Phone: +61414701759

Email: m.moni@unsw.edu.au

\begin{abstract}
Nowadays, cervical cancer (CC) is treated as the leading cancer among women throughout the world. Despite effective vaccination and improved surgery and treatment, $\mathrm{CC}$ remains its fatality rate about half of the infected populations globally. The major screening biomarkers and therapeutic target identification have now become a global concern. The present study, we have employed systems biology approaches to retrieve the potential biomarkers and pathways from the transcriptomic profiling. Initially, we have identified 76 of each up-regulated and down-regulated gene from a total of 4,643 differentially expressed genes. The up-regulatory genes are mainly concentrating on immune-inflammatory response and the down-regulatory genes are on receptor binding and gamma-glutamyltransferase. The involved pathways associated with these genes were also assessed through pathway enrichment and they were mainly focused on different cancer pathways, immunoresponse, and cell cycle pathways. After the subsequent enrichment of these genes, we have identified 12 hub genes, which play a crucial role in CC. Furthermore, the survival of the hub genes was also assessed, and among them, finally, CXCR4 has identified as one of the most potential differentially expressed gene that might play a vital role to the survival of CC patients. Thus CXCR4 could be used as a prognostic biomarker and development of a drug target for CC.
\end{abstract}

Keywords: Systems biology; cervical cancer; prognostic biomarker; differentially expressed genes. 


\section{Introduction:}

Cervical cancer (CC), classified as the second most prominent cancer, is one of the most recurrently diagnosed cancers in terms of prevalence and source of cancer-related deaths in women worldwide [1]. According to World Health Organization (WHO), cervical cancer represents almost $6.6 \%$ of all cancers of females, with an estimated 570,000 new cases in 2018 where low- and middle-income countries experienced an average of 90\% deaths [2-4]. Developed countries have also experienced the fatality of this cancer; for instance, only the USA had accounted for almost 13,170 newly diagnosed cervical cancer cases and 4,250 deaths in 2019 [5].

Human papillomavirus (HPV) infection suggested as the first and foremost cause of cervical cancer pathogenesis [6-8], and studies reported that HPV is responsible for genome aberrations and increases specific target-wise gene expression [9]. Apart from mutations in P53, PIK3CA, PTEN genes, an altered number of oncogenes copies have been reported as the core factor of cervical carcinoma progression [10-12]. Other risk factors such as smoking, pregnancy history, and long term use of oral contraceptives have also been conveyed [13].

The available treatment strategies such as surgery, radiotherapy, chemotherapy are though the prodigious hope for cervical cancer patients, however near about $75 \%$ of patients develop further progression and/ recurrent tumour $[14,15]$. Disease heterogeneity of patients is another challenge to apply a specific treatment method [5].

On the other hand, diagnosis and prognosis of cervical carcinoma are still poorly recognized, insufficient, insensitive, and not specific to in-time or prior identification of the carcinoma properly in the clinical phase [16]. Therefore, there is a pressing need to identify new suitable molecular markers or models to predict the diagnosis/prognosis of cervical cancer.

A series of studies have already been conducted by relying on gene expression patterns to sort out auspicious molecular gene signatures to use as a recurrence prediction tool. A signature of 7-genes series identified in the early stage, [17] and a predictive prognostic model for recurrent tumour had constructed, which is composed of a 12-genes series [18]. Through the analysis of long non-coding RNA (lnc-RNA), it was revealed that 9-genes signature sets were used to predict patients' chance to develop recurrent tumours [19]. However, searching for an effective series of gene sets is to be under investigation that can be used as a promising prognosis and/ diagnosis purpose to mitigate the disease outcome at the very early stage.

Nowadays integrative systems biology approach is a promising technique, which has been applied to predict novel molecular oncogenes and gene signatures using existing gene expression profiles from Gene Expression Omnibus (GEO) [20-26].

In the current research, we have employed systems biology approaches to explore the differentially expressed genes (DEGs), gene network, pathways, and protein-protein interactions unique to $\mathrm{CC}$ to retrieve potential biomarkers and pathways of cervical carcinoma.

\section{Materials and Methods}

The entire procedure of integrative systems biology analytical approach to identify novel molecular gene signatures and pathways of cervical cancer is shown in Figure 1 through the schematic diagram. 


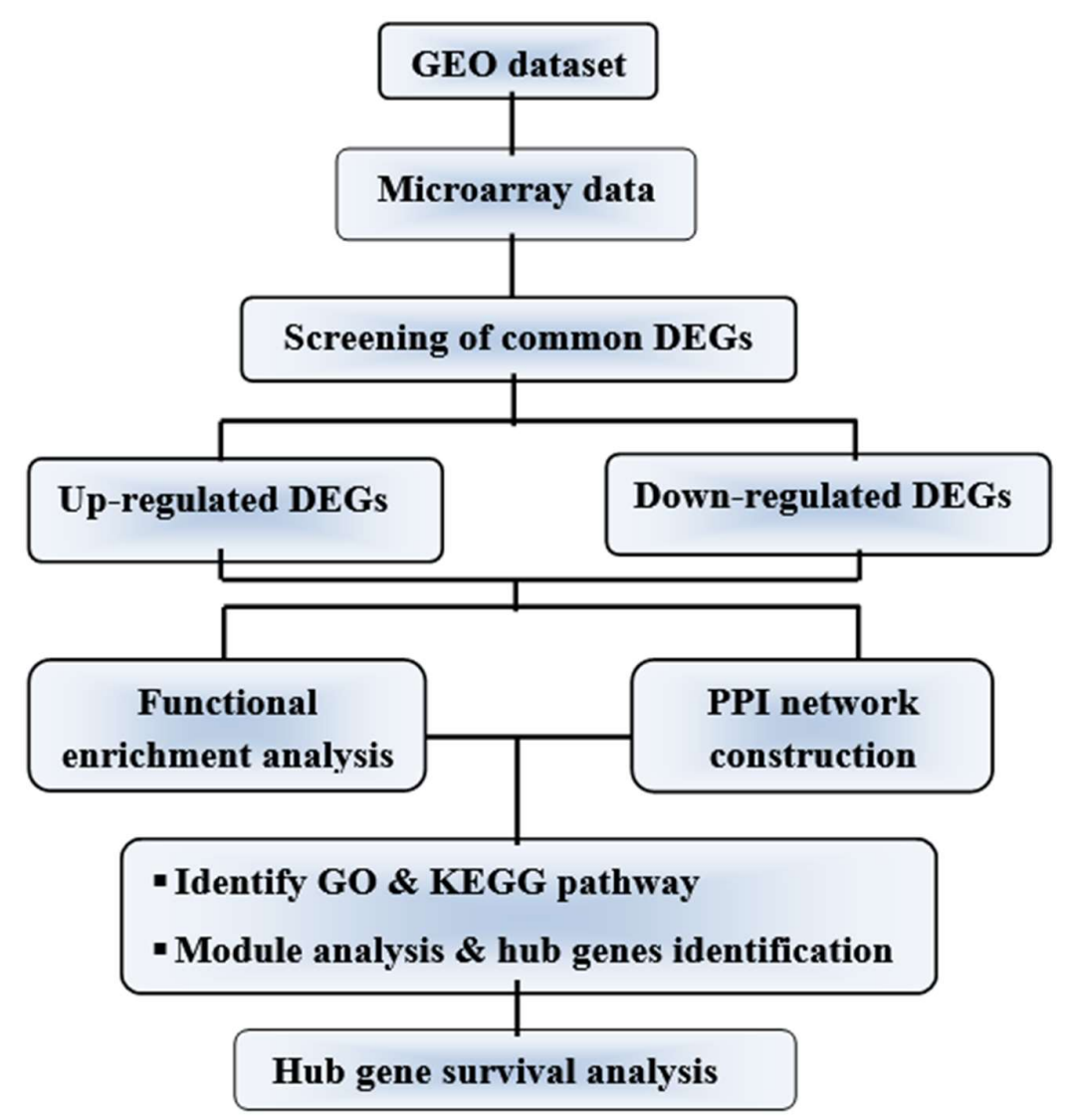

Figure 1: Flow diagram of integrative bioinformatics analytical approach applied in this study.

\subsection{Data Retrieval}

The gene expression profile (GSE148747) of primary fibroblasts from the normal region vs. tumourous region of the human uterine cervix, based on the platform of GPL4133 (Agilent014850 Whole Human Genome Microarray 4x44K G4112F), were collected from the Gene Expression Omnibus (GEO) (https://www.ncbi.nlm.nih.gov/geo/) database, a public repository that provides free access to a full set of microarray, next-generation sequencing and other forms of high-throughput functional genome data submitted by the different research group [27]. A total of eight samples were used in this dataset (GSE148747). The GSM4478163, GSM4478166, GSM4478167 GSM4478168, and GSM4478170 were used as normal primary fibroblast, and the GSM4478164, GSM4478165, and GSM4478169 were used as tumour-associated fibroblast.

\subsection{Screening of Differentially Expressed Genes (DEGs)}

We utilized the GEO2R (http://www.ncbi.nlm.nih.gov/geo/geo2r/) statistical tool to calculate and assess the genes that were expressed differently between the primary fibroblasts from the normal region and tumourous region of the human uterine cervix [28]. The Benjamini and Hochberg (false discovery rate) and t-test methods were utilized with the GEO2R tool to 
calculate the FDR and p-values, respectively, to identify the DEGs [29]. We considered Pvalue $\mathrm{p}<0.05$ and a $\operatorname{logFC}$ (large-scale fold changes) $>1$ to be statistically significant for the DEGs, and a $\log \mathrm{FC} \geq 1, \log \mathrm{FC} \leq-1$ were considered to indicate up-regulated and downregulated DEGs, respectively [30-32]. The resultant DEG dataset was collected and used for further analysis. Including all of the DEGs identified in the samples, we constructed a volcano plot by using the pheatmap package in $\mathrm{R}$ language.

\subsection{Functional Enrichment of Gene Sets}

The initial ontology of gene (GO) and KEGG pathway enrichment analyses of the DEGs were annotated $(p<0.05)$ using the online bioinformatics tool DAVID v6.8 [33]. The human genome was selected as the background parameter, and Affymetrix based identification was selected as identifier. The KEGG pathway enrichment analyses of the DEGs were crosschecked using the NetworkAnalyst online tool [34]. Gene Ontology (GO) study is a frequently used approach for the functional studies of large-scale transcription or genomic data $[35,36]$. Similarly, the Kyoto encyclopedia of genes and genomes (KEGG) is generally used to understand metabolic pathways widely for gene annotation [37, 38].

\subsection{PPI Network Construction}

The online database STRING (v11.0, http://www.string-db.org/) was used to construct the PPI network of the proteins encoded by DEGs. The String is an online repository with 24,584,628 proteins from 5,090 organisms to predict the relationship between genes [39]. The combined score was set at less than 0.75 (medium confidence score) to be considered significant.

\subsection{Selection of Central Hub Proteins from the PPI Network}

The obtained PPI networks were visualized by Cytoscape (http://www.cytoscape.org/) [40]. The Cytoscape plug-in Molecular Complex Detection (MCODE) [41] was applied to obtain significant modules with an established score of greater than 3 and nodes of greater than 4 . In the PPI network, the number of edges involved determines the degree value of the nodes; nodes with high degree values were considered to be hub genes. We mapped the hub genes to evaluate their PPI information. We use cytoHubba [42] (a Cytoscape plugin) to evaluate hub genes from the constructed PPI network. The cytoHubba is a tool that uses 11 specific methods to calculate hub genes from the PPI network; in this study, we use degree score to identify hub genes.

\subsection{Hub gene Survival Analysis}

A comprehensive online platform called Gene Expression Profiling Interactive Analysis (GEPIA2) [43] provides fast and customized delivery of functionalities based on TCGA (The Cancer Genome Atlas) and genotype-tissue expression (GTEx) data. GEPIA2 evaluates the survival effect of differentially expressed genes in a given cancer sample. The overall survival effect of hub genes in $\mathrm{CC}$ was estimated by calculating the log-rank p-value and the HR (hazard ratio-95\% confidence interval) using GEPIA2 single-gene analysis. 


\section{Results and Analysis}

\subsection{DEG Identification}

The expression profiling was performed on the CC gene dataset GSE148747, which was retrieved from GEO and the overall patients' information is shown in Table 1. To identify the DEGs from these two groups, we conducted GEO2R web-server to calculate the p-values and $\log 2 \mathrm{FC}$ values. The resulting genes that met the cutoff criteria $(\log \mathrm{FC} \geq 1, \log \mathrm{FC} \leq-1$, and $\mathrm{P}-$ value $\mathrm{p}<0.05$ ) were considered DEGs. Overall, 4,643 genes were identified from the GEO dataset using the GEO2R tool. The volcano-plot was constructed by comparing the two groups and is depicted in Figure 2. Finally, among them, a total of 152 DEGs were identified based on the cut off criteria $(\operatorname{logFC} \geq 1, \operatorname{logFC} \leq-1$ and an adjusted P-value $\mathrm{p}<0.05)$ where 76 and 76 were up-regulated and down-regulated, respectively (Table S1 and S2).

Table 1: Patients information in GSE148747 derived from the GEO database.

\begin{tabular}{|c|c|c|c|l|}
\hline Group & Accession & Organism & Disease state & \multicolumn{1}{|c|}{ Cell Type } \\
\hline \multirow{4}{*}{ Normal } & GSM4478163 & Homo sapiens & Normal & Normal fibroblast \\
\cline { 2 - 5 } & GSM4478166 & Homo sapiens & Normal & Normal fibroblast \\
\cline { 2 - 5 } & GSM4478167 & Homo sapiens & Normal & Normal fibroblast \\
\cline { 2 - 5 } & GSM4478168 & Homo sapiens & Normal & Normal fibroblast \\
\cline { 2 - 5 } & GSM4478170 & Homo sapiens & Normal & Normal fibroblast \\
\hline Tumor & GSM4478164 & Homo sapiens & Cervical Cancer & $\begin{array}{l}\text { Tumor-associated cervix } \\
\text { fibroblasts }\end{array}$ \\
\cline { 2 - 5 } & GSM4478165 & Homo sapiens & Cervical Cancer & $\begin{array}{l}\text { Tumor-associated cervix } \\
\text { fibroblasts }\end{array}$ \\
\cline { 2 - 5 } & GSM4478169 & Homo sapiens & Cervical Cancer & $\begin{array}{l}\text { Tumor-associated cervix } \\
\text { fibroblasts }\end{array}$ \\
\hline
\end{tabular}

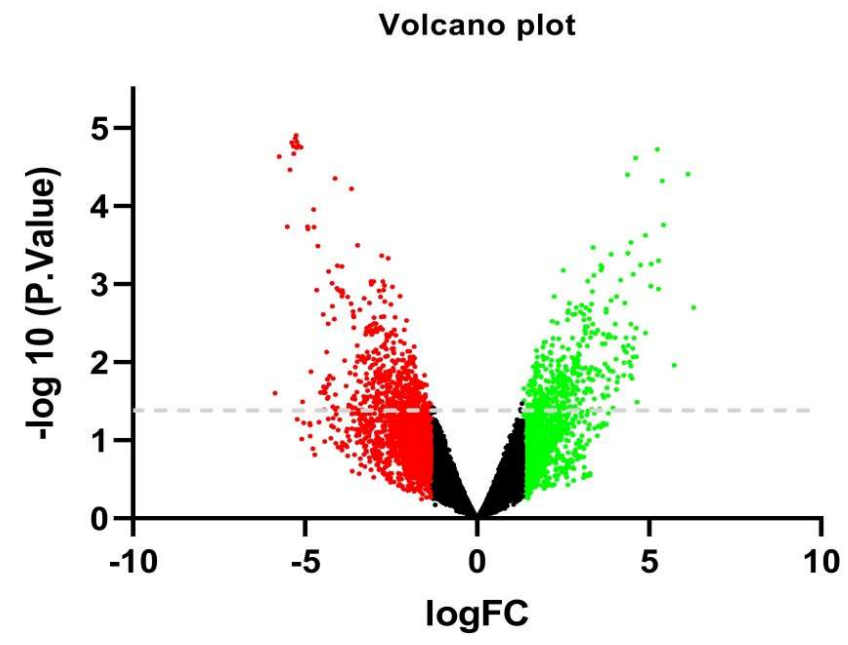

Figure 2: Volcano-plot of the DEGs from the GSE148747 dataset. X-axis: logFC, large-scale fold changes; $y$-axis: $-\log 10$ of the p-value is showing the statistical significance. The green point represents the up-regulated genes where red are the down-regulated genes. 


\subsection{Functional analysis of DEGs}

By using the DAVID database, the top 10 enrichment analysis outcomes were screened for the up-regulated and down-regulated DEGs of the GO analysis. The biological process (BP), cellular component (CC), and molecular function (MF) enrichment analysis outcomes are displayed in Figures 3A and 3B. DAVID and NetworkAnalyst analysis of KEGG pathway outcomes for the significantly enriched DEGs are depicted in Figure 4. The overlapped gene lists of up- and down-regulated genes were involved in GO were shown in supplementary Table S3, S4, and the gene-lists of the KEGG pathway were shown in supplementary Table S5, respectively.

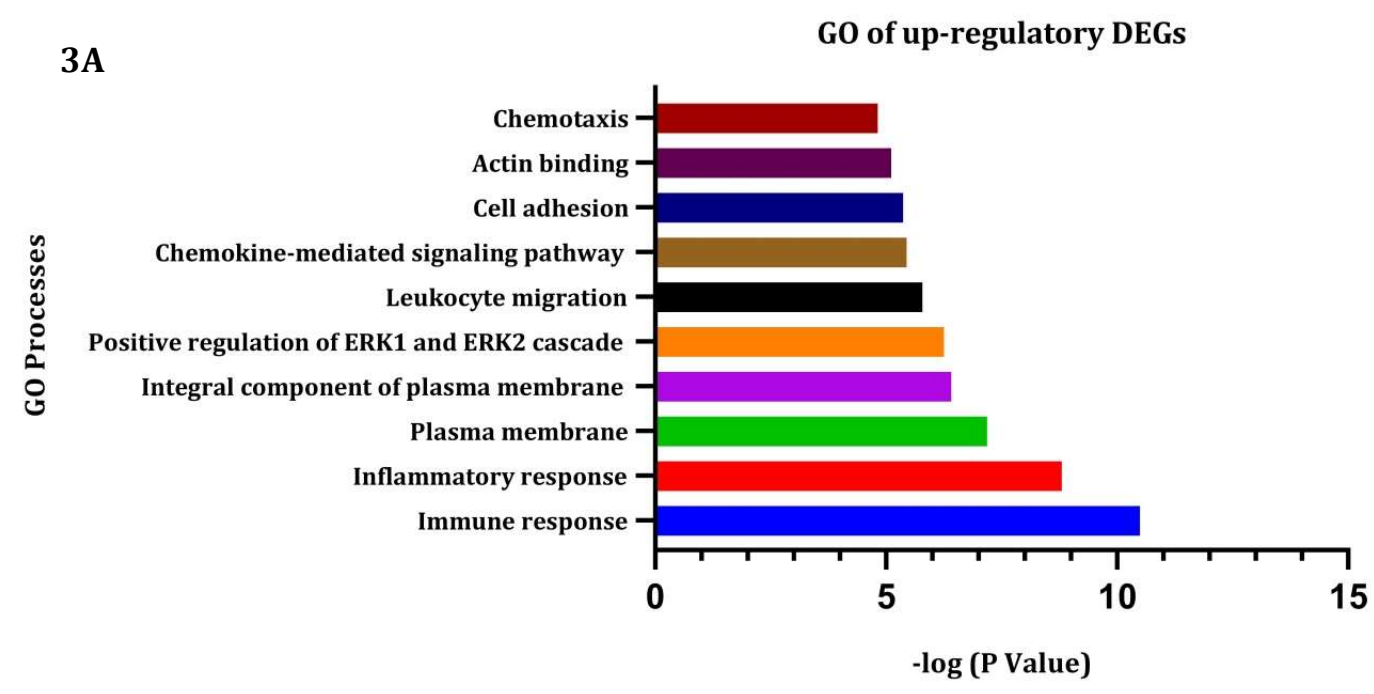

3B

GO of down-regulatory DEGs

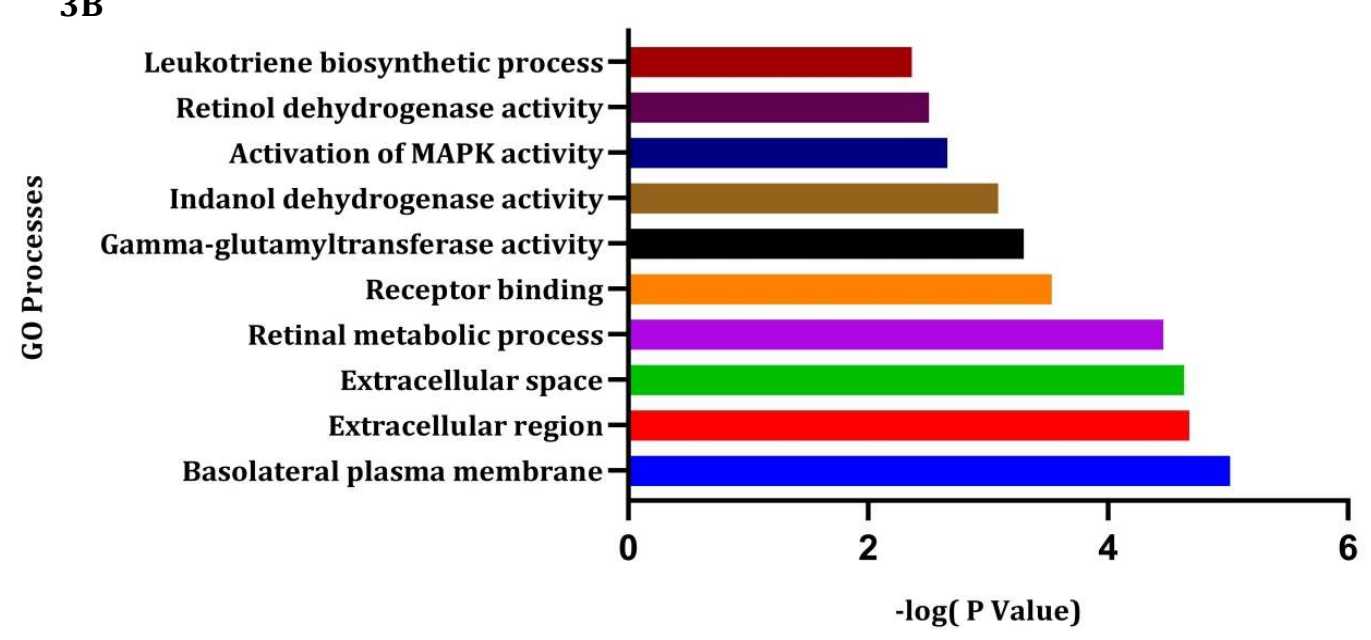

Figure 3: Functional analyses of the differentially expressed genes (DEGs) in cervical cancer. In figure 3A, GO of up-regulatory DEGs and in 3B. GO of down-regulatory DEGs. Each of the GO processes was plotted based on the $-\log (p)$ value. 


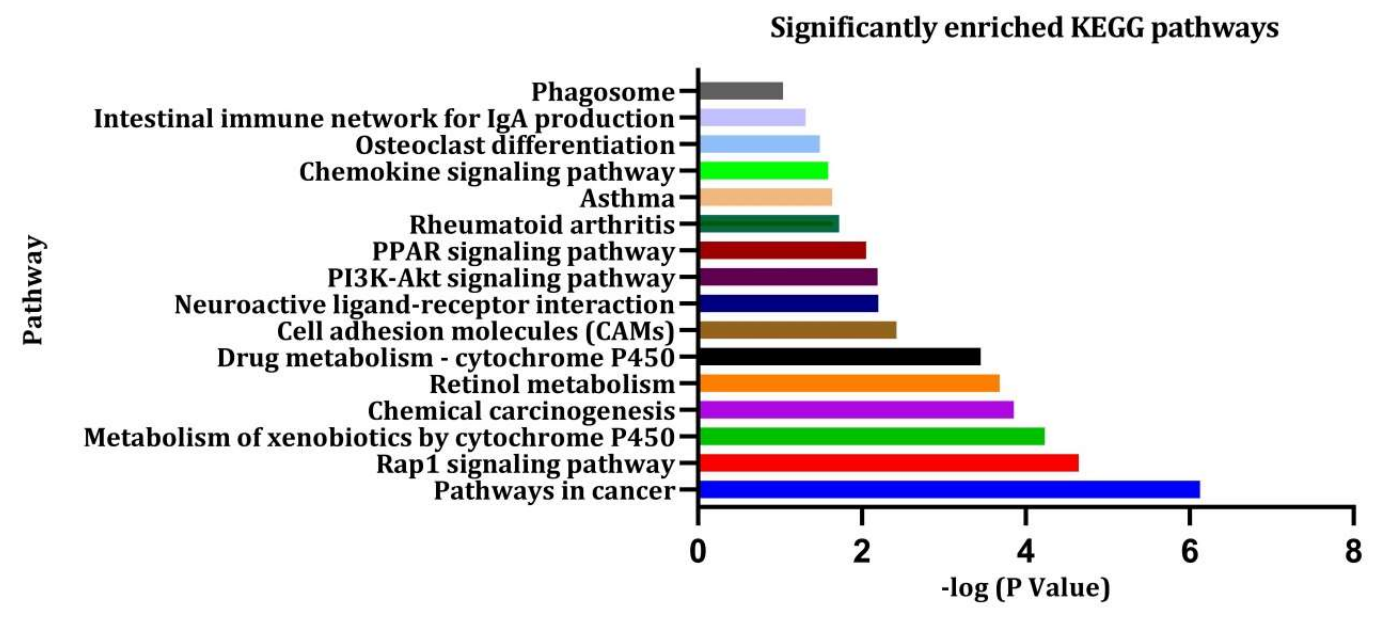

Figure 4: The enriched KEGG pathways generated from the DEGs.

\subsection{PPI Network Construction}

To evaluate the PPIs between the DEGs, we used the STRING tool to identify the PPI networks for both the up- and down-regulated genes. Thereafter, the resulting PPI network from STRING was exported as a ".txt" file and imported as a.csv file into Cytoscape v3.8.0 software for visualization. After subsequent enrichment of each of the networks, we have generated a merged network of PPI consisting of 152 nodes and 1206 edges (Figure S1). The PPI network for up-regulated DEGs had 76 nodes with 656 edges (Figure 5A) and the PPI network for down-regulated DEGs had 76 nodes with 151 edges between the nodes (Figure 5B).

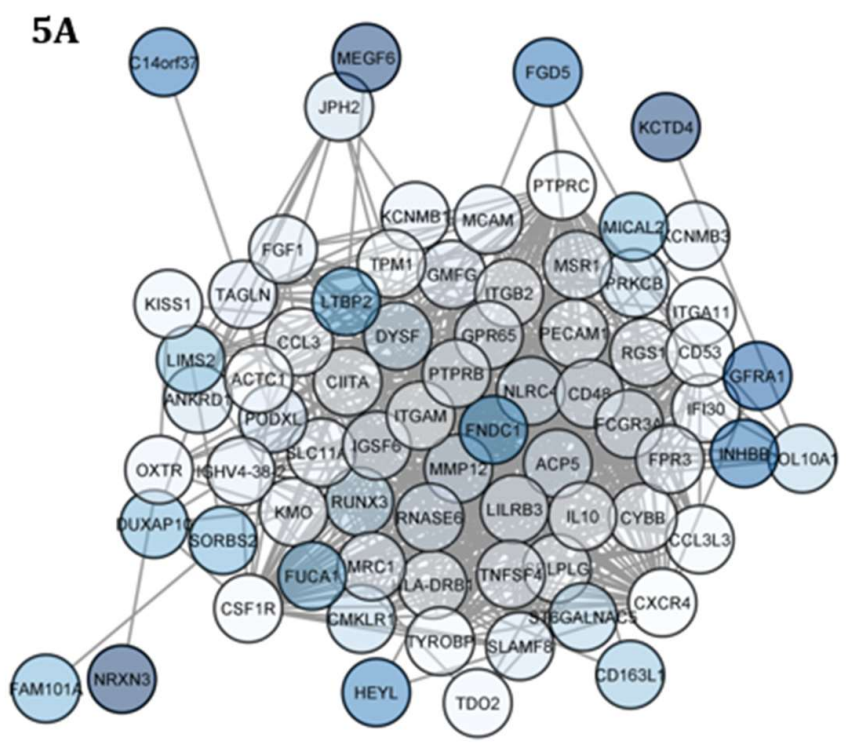




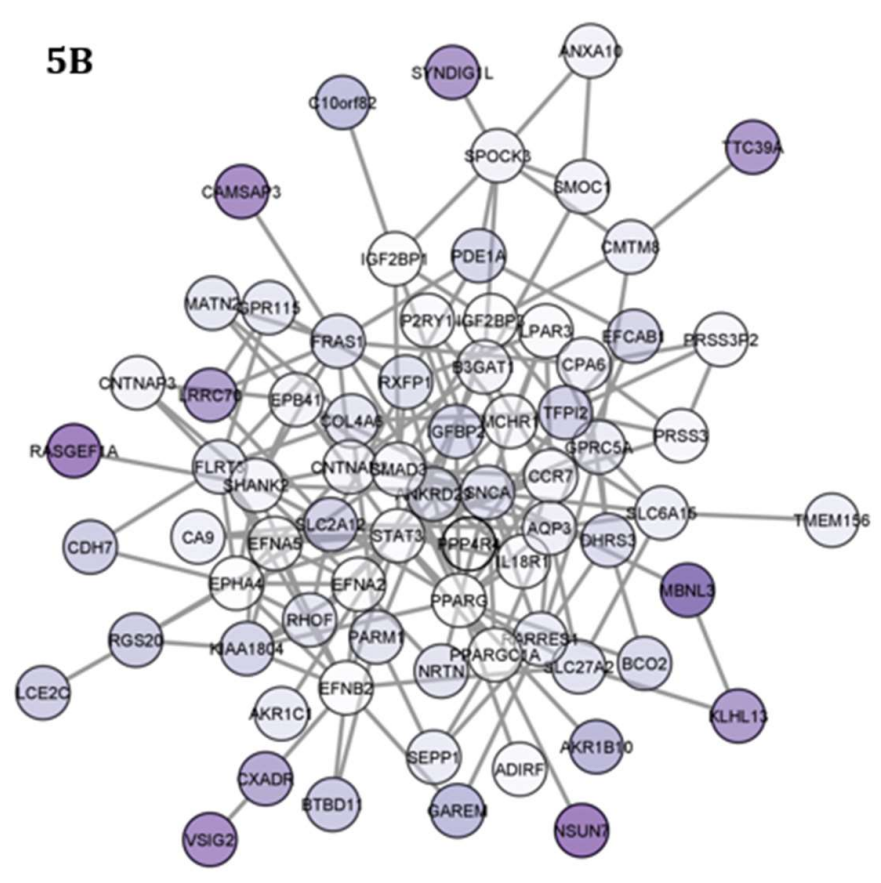

Figure 5: Visualization of the protein-protein interaction network of CC. In figure $5 \mathbf{A}$, the PPI network for up-regulated genes with 76 nodes and 656 edges and in figure 5B the PPI network for down-regulated genes with 76 nodes and 151 edges.

\subsection{Modules and Hub proteins identification}

We identified 3 significant modules from the merged PPI network by using the Cytoscape plugin MCODE. Module 1 had 30 nodes with 187 edges (Figure 6A); on the other hand, Module 2 and 3 had 29 and 24 nodes with 166 and 57 edges, respectively (Figure 6B and 6C). The DEGs of the modules had an important role to enrich significant GO terms 'protein binding' and 'cytoplasm'. We also identified the top 12 hub genes (Table 2) from the PPI network using the cytoHubba tool and are depicted in Figure 7. The topological parameters of the twelve molecular hub genes of the PPI network are also shown in Table 2, including the clustering coefficient, degree, the betweenness centrality, and the closeness centrality for the individual gene.

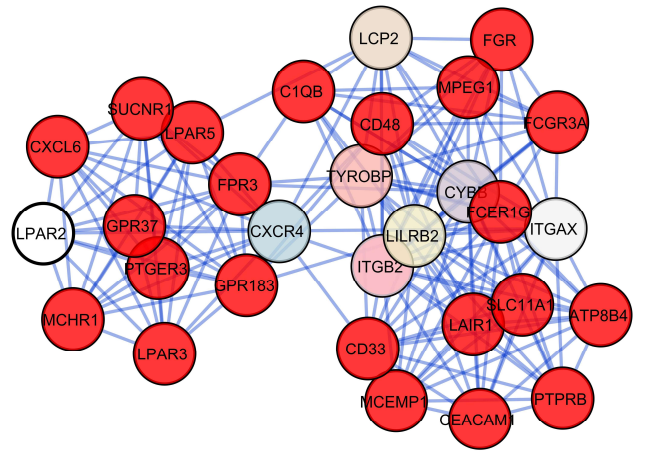

(a)

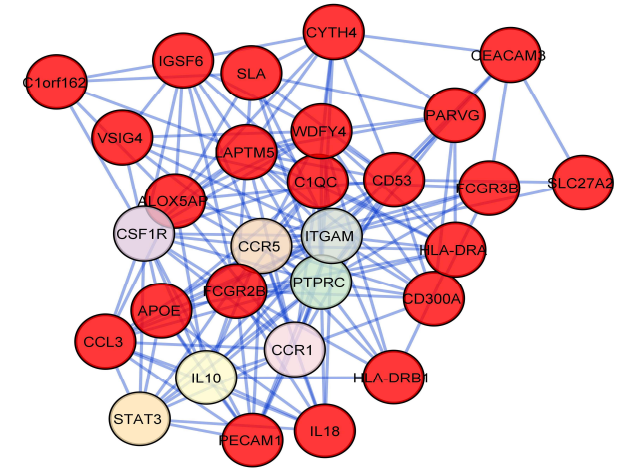

(b) 


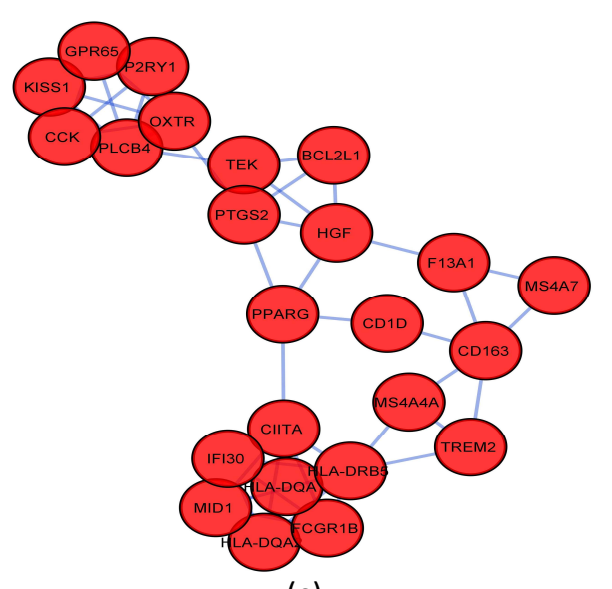

Figure 6: Module analysis of PPI network. A. Module 1 had 30 nodes with 187 edges. B. Module 2 had 29 nodes and 166 edges. C Module 3 had 24 nodes and having 57 edges.

Table 2: Topological parameters for the twelve potential biomarkers of the PPI network.

\begin{tabular}{|c|c|c|c|c|c|}
\hline $\begin{array}{c}\text { Gene Signature } \\
\text { Name }\end{array}$ & Degree & $\begin{array}{c}\text { Betweenness } \\
\text { Centrality }\end{array}$ & $\begin{array}{c}\text { Clustering } \\
\text { Coefficient }\end{array}$ & $\begin{array}{c}\text { Closeness } \\
\text { Centrality }\end{array}$ & Stress \\
\hline PTPRC & 80 & 18133.72223 & 0.23196 & 244.0667 & 167664 \\
\hline ITGAM & 79 & 14447.28068 & 0.23337 & 242.4833 & 140924 \\
\hline IL10 & 70 & 15227.82113 & 0.18841 & 234.9833 & 145074 \\
\hline TYROBP & 69 & 6407.99793 & 0.29113 & 227.9833 & 78988 \\
\hline ITGB2 & 66 & 9280.28036 & 0.28858 & 226.5595 & 95454 \\
\hline CCR5 & 61 & 8309.33294 & 0.29836 & 225.8 & 98026 \\
\hline ITGAX & 60 & 4722.34853 & 0.29492 & 222.35 & 59082 \\
\hline CSF1R & 55 & 7708.90149 & 0.32727 & 221.5333 & 87030 \\
\hline LILRB2 & 55 & 5622.88217 & 0.34007 & 217.1333 & 57172 \\
\hline CXCR4 & 55 & 10433.80294 & 0.24108 & 225.8333 & 95538 \\
\hline STAT3 & 53 & 15091.4144 & 0.20682 & 225.2833 & 126744 \\
\hline CYBB & 50 & 4529.93215 & 0.37469 & 218.95 & 54562 \\
\hline
\end{tabular}




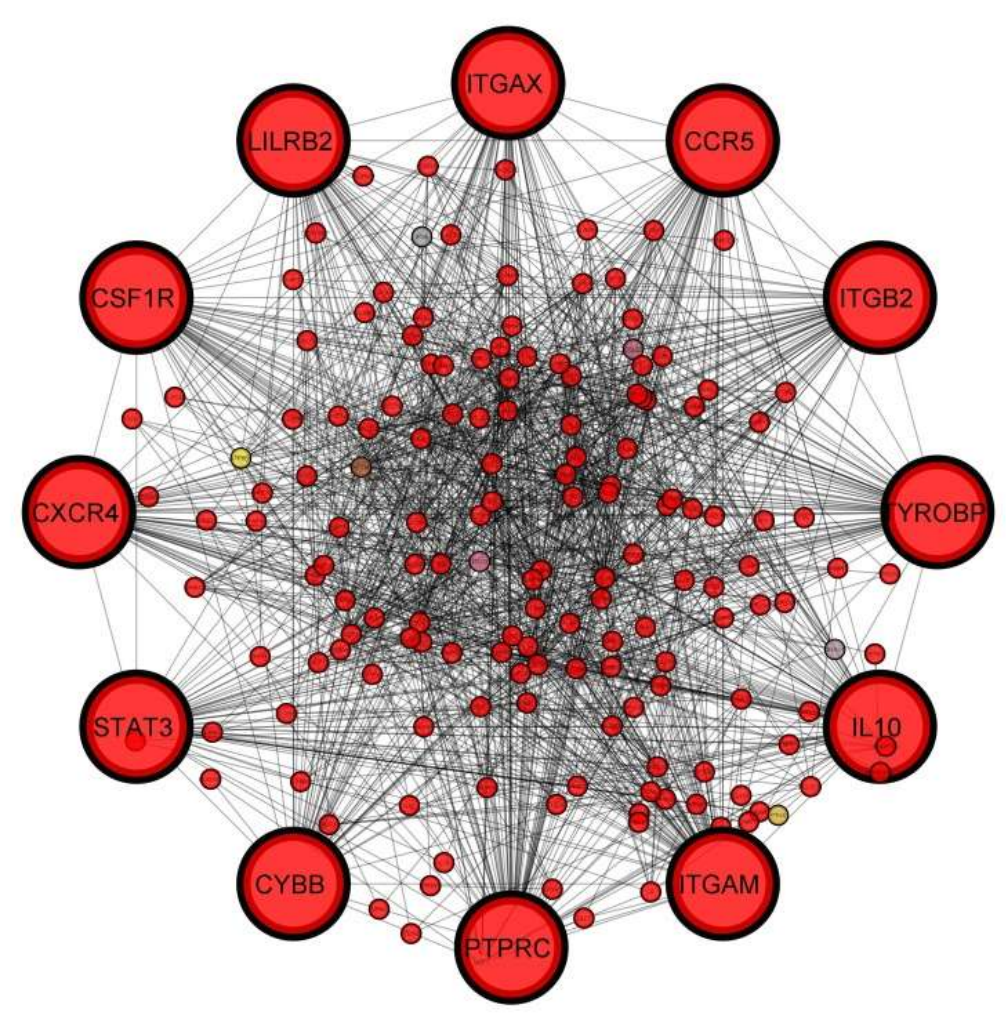

Figure 7: PPI network with identifying hub genes. The highlighted 12 nodes represent the hub genes. The degree score method of cytoHubba considers 94 nodes in this network from the merged PPI network.

\subsection{Survival of the Hub Genes}

GEPIA survival assessment was used to inspect the overall association with survival of 12 hub genes from both the up and down-regulated DEGs and is depicted in Figure 8. Among all the twelve genes, only CXCR4 showed lower overall survival in the higher expression group and having the Hazards Ratio (HR) of 2.6.

\section{Discussion:}

The detection of early-stage CC has an admirable prognosis compared to advanced-stage. The early-stage cases cured significantly through surgery, chemo-radiation, or a blend of treatment approaches, where the advanced level CC patients suffered most because of the often recurrences and incurable nature of CC [1].

The patterns of the DEGs from both tumour and control were visualized by the volcano-plot (Figure 2). The green points represent the up-regulated genes and the red points are the down-regulated genes.

After subsequent enrichment of all the DEGs, we have found some significant up-regulatory GOs such as immune response, inflammatory response, and chemokine-mediated signalling pathway, positive regulation of ERK1 and ERK2 cascade, and chemotaxis in the biological process. For the cellular component, the plasma membrane and integral component of the 
plasma membrane are enriched significantly. The major molecular function up-regulated in the DEGs were immune response and inflammatory response.

On the other hand, down-regulatory GOs such as retinal metabolic process, activation of MAPK activity, and leukotriene biosynthetic process were found in the biological process. The major molecular function down-regulated in the DEGs were receptor binding, gammaglutamyltransferase activity, and indanol dehydrogenase activity.

From the pathway analysis, we have found that most of the pathways were mainly concentrated in different cancer pathways, immunoresponse and cell cycle pathways (Figure 4 and Table S5).

We identified 12 hub genes based on degree value, clustering coefficient, betweenness centrality, and closeness centrality from the merged PPI analysis; these 12 genes PTPRC, ITGAM, IL10, TYROBP, ITGB2, CCR5, ITGAX, CSF1R, LILRB2, CXCR4, STAT3 and CYBB had the diagnostic value which distinguishes $\mathrm{CC}$ from normal individuals (Table 2). A higher percentage of cervical tumours occupied by PTPRC + cells were strongly associated with enhanced tumour-infiltration by Tbet + cells and Foxp3+ cells. Longer disease-free and disease-specific survival were reported in the type I-oriented PTPRC+ cell to infiltrate occupied tumour areas [44]. ITGAM, ITGB2 genes play a pivotal role in cell adhesion in multiple myeloma and reported as potential diagnostic markers [45]. Type-2 cytokine, IL-10 show immunosuppressive functions and capable of stimulating tumour growth and cervical tumour biopsies showed increased presence for mRNA for IL-10 [46]. The chemotactic receptors, CCR5 expressed in CD8 $+\mathrm{T}$ cells which preferentially proliferated in cervical cancer [47], and the proliferation and invasion of cervical cancer can be arrested by downregulating the expression of CCR5 [48]. Similarly, the reduced level of CSF1R protein significantly contributes to suppressing cervical cancer cell proliferation and motility and induces apoptotic cell death [48]. Therefore, CCR5 and CSF1R might be the new targets for cervical cancer treatment. Cervical tumour invades in adjacent tissues and subsequently into distant organs initiated by the expression of CXCR4 through the link of another factor CXCL12 [49]. Also, CXCR4-deficient cells had lower expression of the proliferation marker $\mathrm{Ki}-67$ and decreased ability to engraft into lungs and spleen $[50,51]$. STAT3 accumulation was observed in specific sites especially in basal and suprabasal layers of HPV16-positive early pre-cancer lesions and STAT3 expression and activity were distinctively higher in poorly-differentiated lesions [52]. So, it suggests that the STAT3 gene might be used as a prognostic marker in cervical cancer prognosis at a very primary stage.

Finally, the four genes namely TYROBP, IGTAX, LILRB2, and CYBB have not been previously reported to be associated with cervical cancer. These genes need to be independently validated before they can be useful for evaluating the prognosis and/or diagnosis of CC patients.

According to our predictions, among the identified key 12 hub genes only STAT3 showed the down-regulatory expression patterns whilst all the remained genes are up-regulated but the Kaplan-Meier overall survival analysis denoted that the level of only CXCR4 expression was significantly related to the overall survival of patients with cervical cancer (Figure 8). Importantly, previous studies have been revealed the role of CXCR4 in the regulation of tumour growth. Smith MC et., al has been reported the initiation of cell proliferation and/or survival of cervical cancer cells is not possible without the CXCR4 receptor [53]. Furthermore, the CXCR4 is a very important factor in the metastatic process of cervical cancer and inhibition of CXCR4 expression and function significantly impairs the growth of cervical carcinoma [50]. So, CXCR4 could be a novel target for the prevention of cervical carcinoma growth and metastasis. 
TYROBP
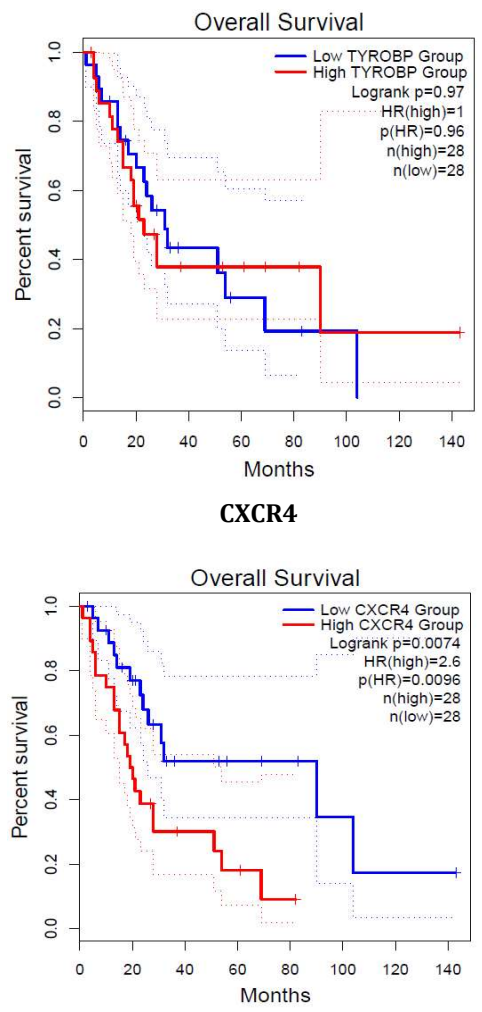

ITGAM

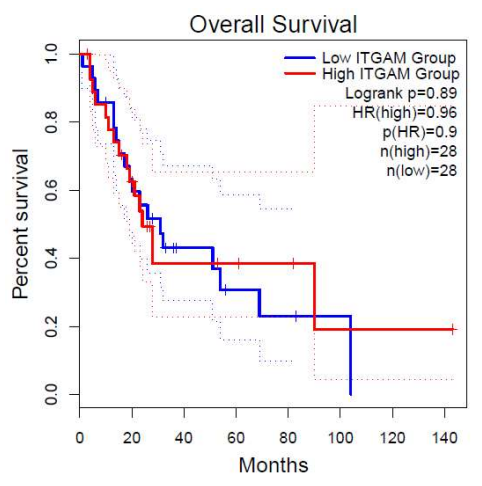

OVk LILRB2

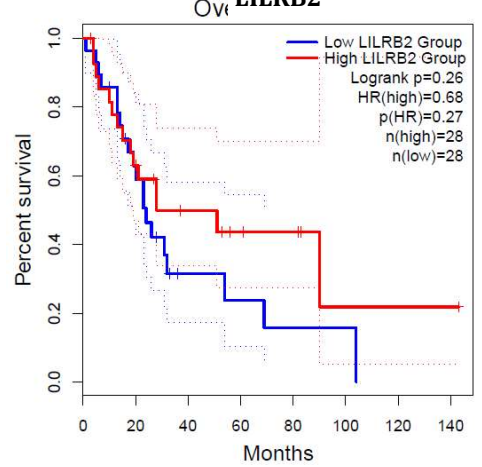

CCR5

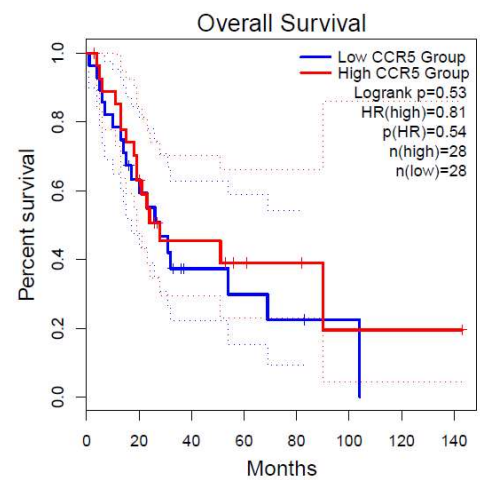

CYBB

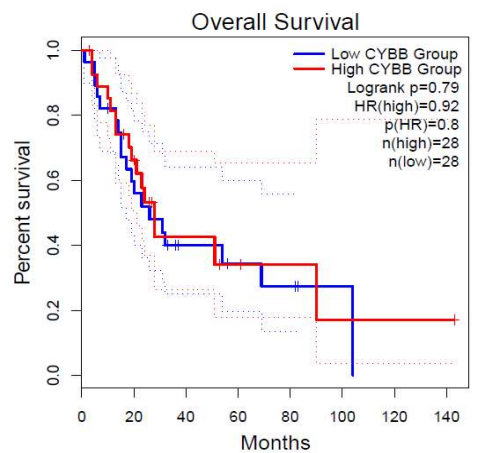

ITGAX

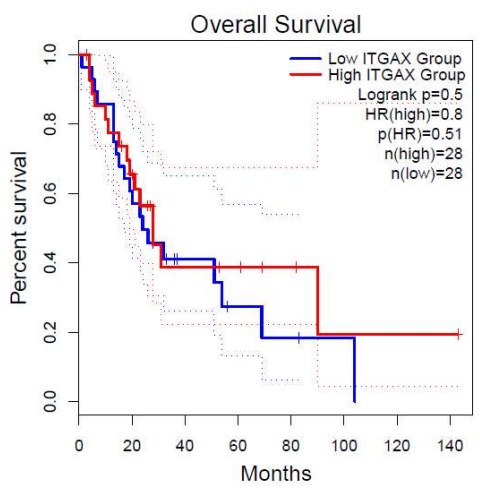

O PTPRC ।

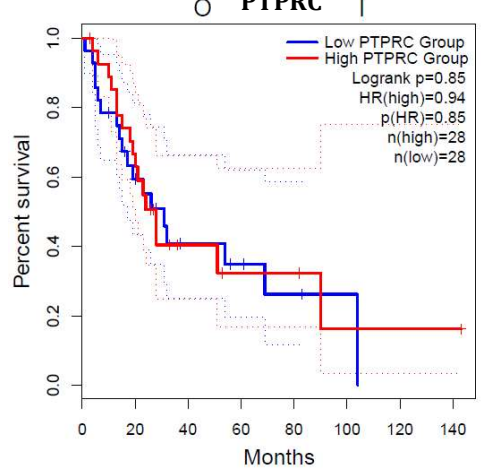

CSF1R

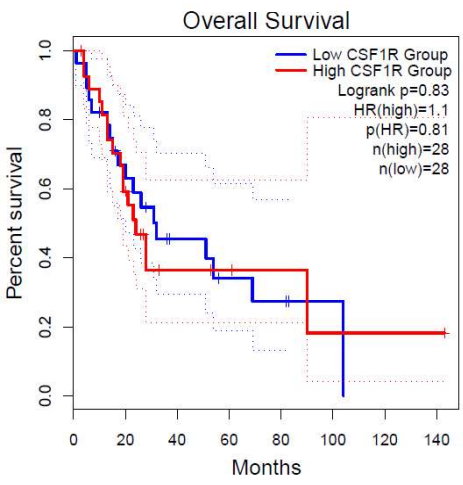

IL10

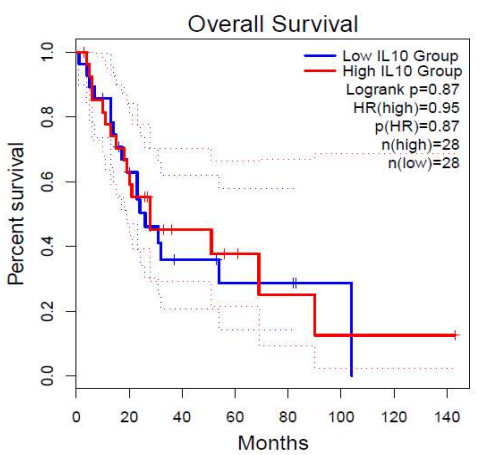

ITGB2
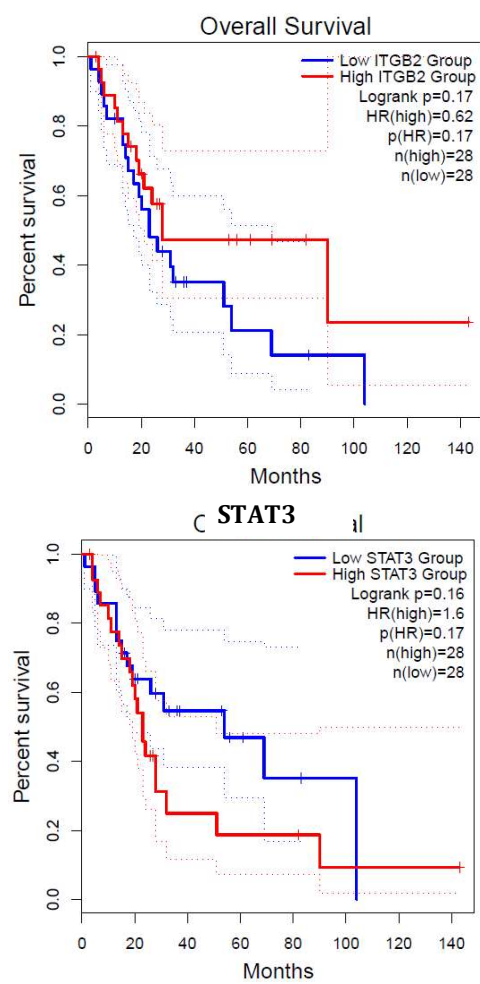

Figure 8 Kaplan-Meier overall survival analysis of the hub genes expressed in CC. 


\section{Conclusion}

In conclusion, our analysis from the current study provides evidence that candidate genes such as PTPRC, CXCR4, CCR5, and CSF1R and their enriched pathways, respectively, of the pathway in the plasma membrane, inflammatory response, and chemokine-mediated signalling might be involved in the pathogenesis of CC. Moreover, CXCR4 might play an important role in the metastasis of CC. We believe that the results obtained above can provide theoretical guidelines for future works in the laboratory. Further wet lab investigation is required to validate and using as a prognostic, diagnostic and/or unique target to support the treatment management of CC.

Declaration of Competing Interests: The authors declare no conflict of interest.

Funding: No funding was received.

Ethical Approval: Not Applicable.

\section{Author contributions}

Arafat Rahman Oany conceived, designed, and guided the study, drafted the manuscript, and analysed the data. Mamun Mia carried out the analysis and drafted the manuscript. Tahmina Pervin drafting the manuscript and helped in analysis. Salem Ali Saleh Alyami and Mohammad Ali Moni participated in coordination, performed critical revision, and helped in drafting the manuscript. All authors read and approved the final manuscript.

\section{References}

1. Bray F, Ferlay J, Soerjomataram I, Siegel RL, Torre LA, Jemal A: Global cancer statistics 2018: GLOBOCAN estimates of incidence and mortality worldwide for 36 cancers in 185 countries. CA: a cancer journal for clinicians 2018, 68(6):394-424.

2. Organization WH: UN Joint Global Programme On Cervical Cancer Prevention and Control. In.; 2017.

3. Ferlay J, Ervik M, Lam F, Colombet M, Mery L, Piñeros M, Znaor A, Soerjomataram I, Bray F: Global cancer Observatory: cancer today. Lyon, France: international agency for research on cancer. Cancer Today 2018.

4. WHO. In: https://wwwwhoint/cancer/prevention/diagnosis-screening/cervical-cancer/en/.

5. Zhao M, Huang W, Zou S, Shen Q, Zhu X: A Five-Genes-Based Prognostic Signature for Cervical Cancer Overall Survival Prediction. International Journal of Genomics 2020, 2020.

6. Walboomers JM, Jacobs MV, Manos MM, Bosch FX, Kummer JA, Shah KV, Snijders PJ, Peto J, Meijer CJ, Muñoz $\mathrm{N}$ : Human papillomavirus is a necessary cause of invasive cervical cancer worldwide. The Journal of pathology 1999, 189(1):12-19.

7. Crosbie EJ, Einstein MH, Franceschi S, Kitchener HC: Human papillomavirus and cervical cancer. The Lancet 2013, 382(9895):889-899.

8. Schiffman M, Castle PE, Jeronimo J, Rodriguez AC, Wacholder S: Human papillomavirus and cervical cancer. The Lancet 2007, 370(9590):890-907.

9. Network CGAR: Integrated genomic and molecular characterization of cervical cancer. Nature 2017, 543(7645):378-384. 
10. Crook T, Wrede D, Tidy J, Vousden K, Tidy J, Mason W, Evans D: Clonal p53 mutation in primary cervical cancer: association with human-papillomavirus-negative tumours. The Lancet 1992, 339(8801):1070-1073.

11. Mclntyre JB, Wu JS, Craighead PS, Phan T, Köbel M, Lees-Miller SP, Ghatage P, Magliocco AM, Doll CM: PIK3CA mutational status and overall survival in patients with cervical cancer treated with radical chemoradiotherapy. Gynecologic oncology 2013, 128(3):409-414.

12. Lee M-S, Jeong M-H, Lee H-W, Han H-J, Ko A, Hewitt SM, Kim J-H, Chun K-H, Chung J-Y, Lee C: PI3K/AKT activation induces PTEN ubiquitination and destabilization accelerating tumourigenesis. Nature communications 2015, 6:7769.

13. Gadducci A, Barsotti C, Cosio S, Domenici L, Riccardo Genazzani A: Smoking habit, immune suppression, oral contraceptive use, and hormone replacement therapy use and cervical carcinogenesis: a review of the literature. Gynecological Endocrinology 2011, 27(8):597604.

14. Kim S-W, Chun M, Ryu H-S, Chang S-J, Kong TW, Lee EJ, Lee YH, Oh Y-T: Salvage radiotherapy with or without concurrent chemotherapy for pelvic recurrence after hysterectomy alone for early-stage uterine cervical cancer. Strahlentherapie und Onkologie 2017, 193(7):534-542.

15. Fuller CD, Wang SJ, Thomas Jr CR, Hoffman HT, Weber RS, Rosenthal DI: Conditional survival in head and neck squamous cell carcinoma: results from the SEER dataset 1973-1998. Cancer: Interdisciplinary International Journal of the American Cancer Society 2007, 109(7):1331-1343.

16. Li X, Tian R, Gao H, Yan F, Ying L, Yang Y, Yang P, Gao Ye: Identification of significant gene signatures and prognostic biomarkers for patients with cervical cancer by integrated bioinformatic methods. Technology in cancer research \& treatment 2018, 17:1533033818767455.

17. Huang L, Zheng M, Zhou Q-M, Zhang M-Y, Yu Y-H, Yun J-P, Wang H-Y: Identification of a 7gene signature that predicts relapse and survival for early stage patients with cervical carcinoma. Medical Oncology 2012, 29(4):2911-2918.

18. Lee Y-Y, Kim T-J, Kim J-Y, Choi CH, Do I-G, Song SY, Sohn I, Jung S-H, Bae D-S, Lee J-W: Genetic profiling to predict recurrence of early cervical cancer. Gynecologic oncology 2013, 131(3):650-654.

19. Mao Y, Dong L, Zheng Y, Dong J, Li X: Prediction of recurrence in cervical cancer using a nine-IncRNA signature. Frontiers in genetics 2019, 10:284.

20. Leite GGF, Scicluna BP, van Der Poll T, Salomão R: Genetic signature related to hemehemoglobin metabolism pathway in sepsis secondary to pneumonia. NPJ systems biology and applications 2019, 5(1):1-9.

21. Rahman MR, Islam T, Zaman T, Shahjaman M, Karim MR, Huq F, Quinn JM, Holsinger RD, Gov $E$, Moni MA: Identification of molecular signatures and pathways to identify novel therapeutic targets in Alzheimer's disease: Insights from a systems biomedicine perspective. Genomics 2020, 112(2):1290-1299.

22. Oany AR, Jyoti TP, Ahmad SAI: An in silico approach for characterization of an aminoglycoside antibiotic-resistant methyltransferase protein from Pyrococcus furiosus (DSM 3638). Bioinformatics and Biology insights 2014, 8:BBI. S14620.

23. Oany AR, Pervin T, Mia M, Hossain M, Shahnaij M, Mahmud S, Kibria K: Vaccinomics approach for designing potential peptide vaccine by targeting Shigella spp. serine protease autotransporter subfamily protein SigA. Journal of immunology research 2017, 2017.

24. Oany AR, Mia M, Pervin T, Hasan MN, Hirashima A: Identification of potential drug targets and inhibitor of the pathogenic bacteria Shigella flexneri $2 a$ through the subtractive genomic approach. In silico pharmacology 2018, 6(1):11.

25. Oany AR, Mia M, Pervin T, Junaid M, Hosen SZ, Moni MAJljoaa: Design of novel viral attachment inhibitors of the spike glycoprotein (S) of severe acute respiratory syndrome 
coronavirus-2 (SARS-CoV-2) through virtual screening and dynamics. International journal of antimicrobial agents 2020, 56(6):106177.

26. Oany AR, Pervin T, Moni MAJl, Genetics, Evolution: Pharmacoinformatics based elucidation and designing of potential inhibitors against Plasmodium falciparum to target importin $\alpha / \beta$ mediated nuclear importation. Infection, Genetics and Evolution 2020:104699.

27. Clough E, Barrett T: The gene expression omnibus database. In: Statistical Genomics. Springer; 2016: 93-110.

28. Ritchie ME, Phipson B, Wu D, Hu Y, Law CW, Shi W, Smyth GK: limma powers differential expression analyses for RNA-sequencing and microarray studies. Nucleic acids research 2015, 43(7):e47-e47.

29. Benjamini Y, Yekutieli D: The control of the false discovery rate in multiple testing under dependency. Annals of statistics 2001:1165-1188.

30. Aubert J, Bar-Hen A, Daudin J-J, Robin S: Determination of the differentially expressed genes in microarray experiments using local FDR. BMC bioinformatics 2004, 5(1):125.

31. Pawitan Y, Michiels S, Koscielny S, Gusnanto A, Ploner A: False discovery rate, sensitivity and sample size for microarray studies. Bioinformatics 2005, 21(13):3017-3024.

32. Islam MR, Ahmed ML, Paul BK, Bhuiyan T, Ahmed K, Moni MA: Identification of the core ontologies and signature genes of polycystic ovary syndrome (PCOS): A bioinformatics analysis. Informatics in Medicine Unlocked 2020:100304.

33. Jiao X, Sherman BT, Huang DW, Stephens R, Baseler MW, Lane HC, Lempicki RA: DAVID-WS: a stateful web service to facilitate gene/protein list analysis. Bioinformatics 2012, 28(13):1805-1806.

34. Xia J, Gill EE, Hancock RE: NetworkAnalyst for statistical, visual and network-based metaanalysis of gene expression data. Nature protocols 2015, 10(6):823.

35. Ashburner M, Ball CA, Blake JA, Botstein D, Butler H, Cherry JM, Davis AP, Dolinski K, Dwight SS, Eppig JT: Gene ontology: tool for the unification of biology. Nature genetics 2000, 25(1):25-29.

36. Hulsegge I, Kommadath A, Smits MA: Globaltest and GOEAST: two different approaches for Gene Ontology analysis. In: BMC proceedings: 2009. Springer: S10.

37. Kanehisa M, Goto S: KEGG: kyoto encyclopedia of genes and genomes. Nucleic acids research 2000, 28(1):27-30.

38. Kanehisa $M$, Sato $Y$, Kawashima $M$, Furumichi $M$, Tanabe M: KEGG as a reference resource for gene and protein annotation. Nucleic acids research 2016, 44(D1):D457-D462.

39. Szklarczyk D, Franceschini A, Wyder S, Forslund K, Heller D, Huerta-Cepas J, Simonovic M, Roth A, Santos A, Tsafou KP: STRING v10: protein-protein interaction networks, integrated over the tree of life. Nucleic acids research 2015, 43(D1):D447-D452.

40. Shannon P, Markiel A, Ozier O, Baliga NS, Wang JT, Ramage D, Amin N, Schwikowski B, Ideker T: Cytoscape: a software environment for integrated models of biomolecular interaction networks. Genome research 2003, 13(11):2498-2504.

41. Bader GD, Hogue CW: An automated method for finding molecular complexes in large protein interaction networks. BMC bioinformatics 2003, 4(1):2.

42. Chin $\mathrm{C}-\mathrm{H}$, Chen $\mathrm{S}-\mathrm{H}, \mathrm{Wu} \mathrm{H}-\mathrm{H}, \mathrm{Ho} \mathrm{C}-\mathrm{W}$, Ko M-T, Lin C-Y: cytoHubba: identifying hub objects and sub-networks from complex interactome. BMC systems biology 2014, 8(S4):S11.

43. Tang Z, Li C, Kang B, Gao G, Li C, Zhang Z: GEPIA: a web server for cancer and normal gene expression profiling and interactive analyses. Nucleic acids research 2017, 45(W1):W98W102.

44. Gorter A, Prins F, van Diepen M, Punt S, van der Burg SHJJotm: The tumour area occupied by Tbet+ cells in deeply invading cervical cancer predicts clinical outcome. Journal of translational medicine 2015, 13(1):1-10. 
45. Peng $\mathrm{Y}, \mathrm{Wu} \mathrm{D}$, Li F, Zhang $\mathrm{P}$, Feng $\mathrm{Y}, \mathrm{He}$ AJCCl: Identification of key biomarkers associated with cell adhesion in multiple myeloma by integrated bioinformatics analysis. Cancer Cell International 2020, 20(1):1-16.

46. Bhairavabhotla RK, Verma V, Tongaonkar H, Shastri S, Dinshaw K, Chiplunkar S: Role of IL-10 in immune suppression in cervical cancer. 2007.

47. Domingos-Pereira S, Decrausaz L, Derré L, Bobst M, Romero P, Schiller JT, Jichlinski P, Nardelli-Haefliger DJMi: Intravaginal TLR agonists increase local vaccine-specific CD8 T cells and human papillomavirus-associated genital-tumour regression in mice. Mucosal immunology 2013, 6(2):393-404.

48. Che L-F, Shao SF, Wang LXJE, medicine t: Downregulation of CCR5 inhibits the proliferation and invasion of cervical cancer cells and is regulated by microRNA-107. Experimental and therapeutic medicine 2016, 11(2):503-509.

49. Huang Y, Zhang J, Cui Z-M, Zhao J, Zheng YJCjoc: Expression of the CXCL12/CXCR4 and CXCL16/CXCR6 axes in cervical intraepithelial neoplasia and cervical cancer. Chinese journal of cancer 2013, 32(5):289.

50. Sekuła M, Miekus K, Majka MJljoo: Downregulation of the CXCR4 receptor inhibits cervical carcinoma metastatic behavior in vitro and in vivo. International journal of oncology 2014, 44(6):1853-1860.

51. Yadav SS, Prasad SB, Das M, Kumari S, Pandey LK, Singh S, Pradhan S, Narayan GJBri: Epigenetic silencing of CXCR4 promotes loss of cell adhesion in cervical cancer. BioMed research international 2014, 2014.

52. Chen C-L, Hsieh F-C, Lieblein J, Brown J, Chan C, Wallace J, Cheng G, Hall B, Lin JJBjoc: Stat3 activation in human endometrial and cervical cancers. British journal of cancer 2007, 96(4):591-599.

53. Smith MC, Luker KE, Garbow JR, Prior JL, Jackson E, Piwnica-Worms D, Luker GDJCr: CXCR4 regulates growth of both primary and metastatic breast cancer. Cancer research 2004, 64(23):8604-8612. 